\title{
Nucleon Spin And Structure Studies With COMPASS
}

\author{
Stephane Platchkov \\ CERN, Geneva, Switzerland \\ and \\ CEA-Saclay, DSM/DAPNIA, Gif-sur-Yvette, France
}

\begin{abstract}
The COMPASS experiment at CERN investigates nucleon structure and spectroscopy with high-intensity muon and hadron beams. Between 2002 and 2004 COMPASS has mainly concentrated on the spin structure of the nucleon via deep-inelastic scattering of polarized muons on polarized nucleons. First results include measurements of the gluon contribution to the nucleon spin via charm production and high- $\mathrm{p}_{\mathrm{t}}$ pairs, $\Lambda$ polarization, vector-meson production, Collins and Sivers asymmetries, inclusive and semi-inclusive deep-inelastic scattering asymmetries and a search for pentaquark states. A short overview of these results is presented.
\end{abstract}

Keywords: Quarks, gluons, nucleon spin, polarization, transversity.

PACS: $24.85 .+\mathrm{p}$

\section{INTRODUCTION}

The COMPASS experiment at CERN was designed for the investigation of hadron structure and spectroscopy with both muon and hadron beams. The main physics observables studied with muon beam are the contribution of the nucleon constituentsquarks and gluons-to the nucleon spin, the flavor-separated spin-distribution functions and the relatively new and yet poorly known transverse spin functions that complete our QCD description of the nucleon. Studies on vector meson production, spin transfer in $\Lambda$-hyperon polarization, or pentaquarks searches are also being carried out.

With hadron beams the physics objectives are pion and kaon polarizabilities, diffractive production of exotic states, search for glueballs and production of double charmed baryons.

During the years 2002-2004 a large amount of data was collected mostly using a muon beam. Only at the end of 2004, three weeks of beam time were dedicated to a preliminary ("pilot") data taking with a hadron beam. In this talk a quick overview on the most significant COMPASS results available today will be given; more detailed discussions on some of the results are presented elsewhere at this conference.

\section{THE COMPASS SET-UP}

The COMPASS set-up was designed for beam energies of 100 to $200 \mathrm{GeV}$ and physics processes in which in addition to the scattered beam particle, one or more outgoing particles are detected. In order to insure a broad dynamic range and a large 
angular acceptance, the set-up is built around two large dipole magnets that define two consecutive spectrometers, suitable for large and small scattering angles respectively. Depending on the required space resolution, counting rates and phase space that has to be covered, various tracking detectors are used. Silicon and scintillating fibers detectors cover the beam region. For small angle scattering and near the beam two types of micromesh detectors (Micromegas and GEM) were developed especially for COMPASS. At large angles and for distances far from the beam, large proportional chambers, or drift detectors (drift or straw chambers) are used. Particle identification is performed using a RICH counter and both electromagnetic and hadron calorimeters.

During the muon data taking a large polarized target, consisting of two oppositely polarized cells, $60 \mathrm{~cm}$ long each, surrounded by a large solenoid, was used. The two cells were filled with a ${ }^{6} \mathrm{LiD}$ target material, for which polarizations better than $50 \%$ are routinely achieved. Data were taken with both longitudinally and transversely polarized target, for about $78 \%$ and $22 \%$ of the running time, respectively.

\section{PHYSICS RESULTS}

Compass has collected data in 2002, 2003 and 2004. The data from the first two years are now analyzed; unless indicated, the results discussed below are from this period.

\section{Quark Contribution To The Nucleon Spin}

Data on the $g_{1}^{d}(x)$ structure function were measured[1] for values of $x$ ranging from 0.004 to 0.7 in the region $1(\mathrm{GeV} / \mathrm{c})^{2}<Q^{2}<100(\mathrm{GeV} / \mathrm{c})^{2}$. The new data are in good agreement with previous results and, in addition, significantly improve the available statistics in the lower part of the $x$, between $x=0.004$ and $x=0.03$. A nextto-leading order global analysis to the world $g_{1}(\mathrm{x})$ data available prior to 2005 was performed. The singlet axial charge $\mathrm{a}_{0}$ was determined at $\mathrm{Q}^{2}=3 \mathrm{GeV}^{2}$. In the $\overline{M S}$ scheme the results indicate values of the quark spin content $\Delta \Sigma$ equal to $0.25 \pm 0.03$ and $0.22 \pm 0.02$ without and with the COMPASS data respectively, indicating a reduction in the experimental uncertainty by $50 \%$.

\section{Flavor Spin Distributions}

Flavor spin distributions are determined through measurements of semi-inclusive deep-inelastic scattering spin asymmetries in which, in addition to the scattered muon, pions or kaons are detected. Due to the use of an isoscalar target COMPASS is able to access the $\Delta u+\Delta d, \Delta \bar{u}+\Delta \bar{d}$ and $\Delta s+\Delta \bar{s}$ distributions. Full separation requires combination of the deuteron with proton target data. Preliminary results for experimental positive and negative hadron asymmetries are now available. The data extend to values of $x$ as low as 0.003 and are in good agreement with the previous measurements. The data also significantly improve the statistical accuracy at low $x$. 


\section{Collins And Sivers Asymmetries}

The transversity distributions $\Delta_{\mathrm{T}} \mathrm{q}(\mathrm{x})$ of the quarks in the nucleon are accessed via semi-inclusive deep-inelastic scattering with transversely polarized target. The Collins asymmetry probes the transverse spin distribution, the Sivers asymmetry is sensitive to the intrinsic transverse momentum of the struck quark. First results from our 2002 deuteron data[2] indicate that both asymmetries are compatible with 0 , contrary to the proton data from HERMES[3]. An explanation for our results[4] could come from the cancellation of the $u$ and $d$ quark flavors, due to the use of an isoscalar target. Further information is expected from our 2003 and 2004 data and to the use of a proton target in 2006. Two additional channels leading to the determination of $\Delta_{\mathrm{T}} \mathrm{q}(\mathrm{x})$ are also being investigated: longitudinal $\Lambda$-polarization and two-hadron asymmetry[5].

\section{Gluon Contribution To The Nucleon Spin}

Two different methods are used[6] to access the gluon contribution to the nucleon spin, both being based on the Photon-Gluon Fusion (PGF) process. In the first method (high- $\mathrm{p}_{\mathrm{T}}$ pairs) hadron pairs with opposite charges and large transverse momenta $\left(p_{T, 1}^{2}+p_{T, 2}^{2}>2.5 \mathrm{GeV}^{2}\right)$ are detected. This method has good statistical accuracy but relies on theoretical assumptions on the magnitude of the background processes. In the second method (open charm) detection of the charmed $\mathrm{D}^{0}$ and $\mathrm{D}^{+*}$ mesons via the decay $\mathrm{D}^{0} \rightarrow \mathrm{K}+\pi$ in the final state, signs the PGF process. Using the first method, data were analyzed[7] for $\mathrm{Q} 2>1 \mathrm{GeV}^{2}$ and for $\mathrm{Q}^{2}<1 \mathrm{GeV}^{2}$. In both kinematics the results are in good agreement, the statistical error being better for $\mathrm{Q}^{2}<1 \mathrm{GeV}^{2}$. The resulting value of the gluon polarization is: $\Delta \mathrm{G} / \mathrm{G}\left(x_{\mathrm{g}}=0.1\right)=0.024 \pm 0.089$ (stat) \pm 0.057 (syst).

\section{PERSPECTIVES}

The results available today (see also Refs.7-8) illustrate the important physics potential of COMPASS. Most of the results include 2002/2003 data only. The data from 2004 should double the statistics. After a shutdown in 2005, data taking will resume in 2006. Major improvements of the spectrometer have been undertaken: new solenoid magnet, upgraded RICH read-out system, full electro-magnetic calorimeter coverage, etc... All these modifications will further expand the possibilities of our spectrometer and should improve the $\Delta \mathrm{G} / \mathrm{G}$ factor of Merit by more than a factor of 2 .

\section{REFERENCES}

1. COMPASS Collaboration, E. S. Ageev et al., Physics Letters B612 154-164 (2005).

2. COMPASS Collaboration, V. Yu. Alexakhin et al., Physical Review Letters 94202002 (2005).

3. A. Airapetian et al., Physical Review Letters 94, 012002 (2005).

4. A. Bressan, talk at this conference.

5. R. Joosten, talk at this conference

6. J. Nasalski, talk at this conference.

7. COMPASS Collaboration, hep-ex/0511028, accepted for publication in Physics Letters $\boldsymbol{B}$.

8. A. Szandacz, talk at this conference

9. J. Friedrich, Talk at this conference 